\title{
Mitochondrial quality control and
} neurological disease:

\section{an emerging connection}

\section{Inês Pimenta de Castro ${ }^{1,2,3}$, L. Miguel Martins ${ }^{1, *}$ and Roberta Tufi ${ }^{1}$}

The human brain is a highly complex organ with remarkable energy demands. Although it represents only $2 \%$ of the total body weight, it accounts for $20 \%$ of all oxygen consumption, reflecting its high rate of metabolic activity. Mitochondria have a crucial role in the supply of energy to the brain. Consequently, their deterioration can have important detrimental consequences on the function and plasticity of neurons, and is thought to have a pivotal role in ageing and in the pathogenesis of several neurological disorders. Owing to their inherent physiological functions, mitochondria are subjected to particularly high levels of stress and have evolved specific molecular quality-control mechanisms to maintain the mitochondrial components. Here, we review some of the most recent advances in the understanding of mitochondrial stress-control pathways, with a particular focus on how defects in such pathways might contribute to neurodegenerative disease.

Mitochondria are energy-converting organelles that are present in the cells of virtually all eukaryotic organisms. These cellular powerhouses use oxygen to harness energy through the oxidative phosphorylation (OXPHOS) process. To achieve this, high-energy electrons derived from the oxidation of food molecules are transferred along the respiratory chain (RC) to four multisubunit protein complexes embedded in the inner mitochondrial membrane, releasing energy that is used to pump protons across this membrane. The established proton gradient and resulting mitochondrial membrane potential $\left(\Delta \psi_{\mathrm{m}}\right)$ are then used by adenosine triphosphate (ATP) synthetase to generate ATP, which provides the cell with the majority of its energy requirements. This process of energy production was developed by bacteria approximately two billion years ago, before the appearance of eukaryotic cells. According to the endosymbiotic theory, mitochondria were once

\footnotetext{
${ }^{1}$ Cell Death Regulation Laboratory, MRC Toxicology Unit, Lancaster Road, Leicester LE1 9HN, UK. ${ }^{2}$ Cancer Biology Group, IPATIMUP- Institute of Molecular Pathology and Immunology of the University of Porto, University of Porto, Portugal.

${ }^{3}$ Department of Microbiology, Faculty of Pharmacy, University of Porto, Portugal.

*Corresponding author: L. Miguel Martins, Cell Death Regulation Laboratory, MRC Toxicology Unit, University of Leicester, Hodgkin Building, Lancaster Road, Leicester LE1 9HN, UK. E-mail: lmm24@le.ac.uk
}

Accession information: doi:10.1017/S1462399410001456; Vol. 12; e12; April 2010 (c) Cambridge University Press 2010. Re-use permitted under a Creative Commons Licence - by-nc-sa. 
aerobic bacteria that united with the ancestor of the eukaryotic cell in a mutually advantageous relationship, and this led to an evolutionary explosion from which multicellular organisms evolved (Ref. 1).

Interestingly, mitochondria exist as an intracellular network, and constantly merge through fusion and divide through fission. Fusion consists of the joining of separate mitochondria and is mainly controlled by GTPases. In mammals, mitofusins (MFN1 and MFN2) mediate the fusion of the outer membrane of two mitochondria, followed by the joining of their inner membranes mediated by OPA1 (optic atrophy 1) (Fig. 2). By contrast, DNM1L (dynamin-1 like protein; DRP1) and FIS1 (fission 1 homologue) are key components of the fission machinery, which in cooperation, lead the splitting of the mitochondrial tubule. These two opposing processes are finely balanced, thus maintaining steady state physiological conditions, and have important roles in mitochondrial function and development, as well as in programmed cell death (for a review, see Ref. 2).
Although the majority of the estimated 1500 proteins present in the mitochondrion (Ref. 3) are encoded by the nuclear genome, mitochondria have their own DNA within the matrix, which encodes a small set of mitochondrial proteins - 13 subunits of the RC complexes - as well as rRNAs and tRNAs. In mammalian cells, mitochondrial DNA (mtDNA) is a circular molecule that is present in several copies and is organised into bacterial nucleoid-like structures. These structures contain several proteins that are involved in mtDNA maintenance and replication, as well as in its transcription and translation (Ref. 4). The physical proximity of the nucleoids to the RC makes mtDNA particularly vulnerable to damage by reactive oxygen species (ROS), which are unavoidable OXPHOS products. Accordingly, mitochondria have quality-control systems in place to maintain their function, and they selectively respond to oxidative stress through mechanisms acting at the molecular, organellar and cellular levels (Figs 1 and 2). At the molecular level, the first line of protection is

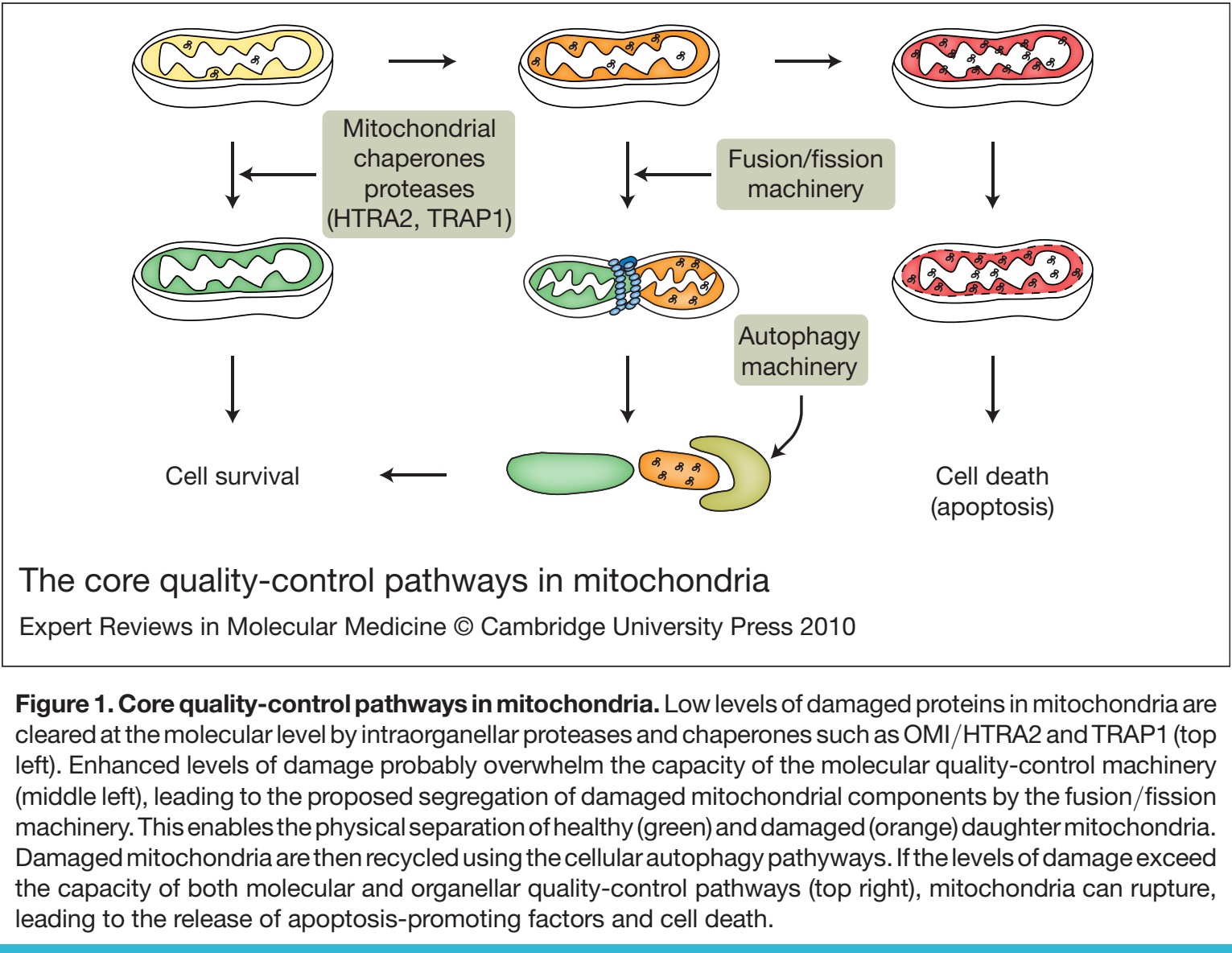

Accession information: doi:10.1017/S1462399410001456; Vol. 12; e12; April 2010 (C) Cambridge University Press 2010. Re-use permitted under a Creative Commons Licence - by-nc-sa. 


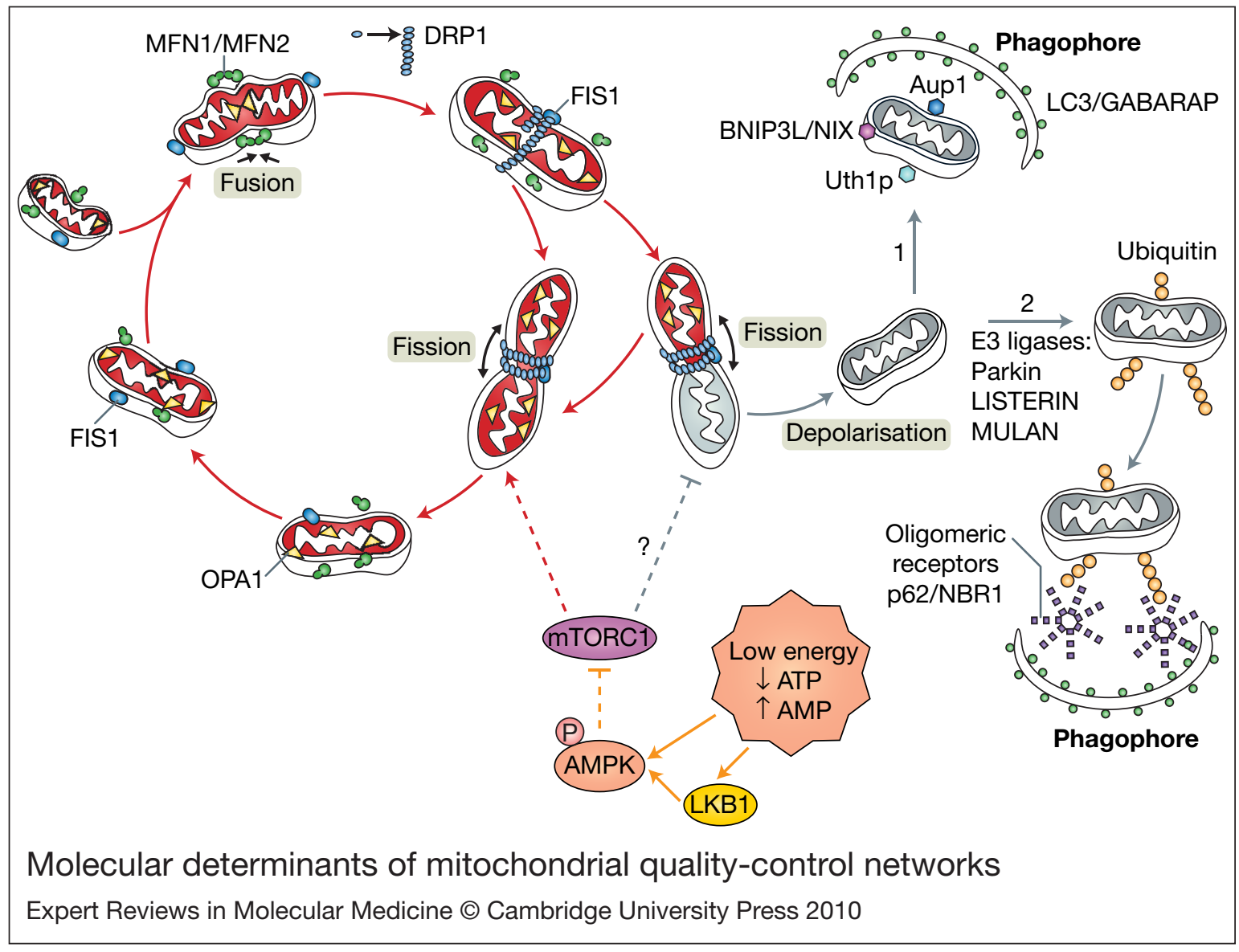

Figure 2. Molecular determinants of mitochondrial quality-control networks. Mitochondria are dynamic organelles that are continuously remodelled by fusion and fission events. Fusion requires the joining of both the inner and outer mitochondrial membranes of two mitochondria. Mitofusin 1 (MFN1) and mitofusin 2 (MFN2) carry out the initial joining of the outer membrane fusion, whereas OPA1 mediates the fusion of the inner membrane. Mitochondrial fission is mediated by DRP1 and FIS1; DRP1 oligomerizes into 8-12 subunit chains and is recruited to mitochondria via the adaptor FIS1. This recruitment allows the full oligomerisation of DRP1 into spiral chains that wrap around the mitochondria and, by constriction, causes mitochondrial fission. In this figure, fission is shown as a means to selectively eliminate damaged mitochondrial components by packing them in one of the fission products, and it has been proposed to involve the asymmetrical segregation of OPA1 (Ref. 5). In this context, fission produces metabolically different daughter units that may either maintain (red) or lose (grey) an intact membrane potential. Depolarised mitochondria are targeted to degradation by autophagy (mitophagy) through a mechanism that is still poorly defined. Here, we illustrate two possibilities: (1) the presence of proteins associated with mitochondrial membranes, such as the mammalian BNIP3L/NIX (Ref. 109, 110), and the yeast proteins Uth1p (Ref. 111) and Aup1 (Ref. 112), which are involved in the direct entrapment of mitochondria by autophagosomes; (2) the activity of E3 ligases, such as LISTERIN (Ref. 113), MULAN (Ref. 114) and Parkin (Refs 28,115$)$, which might regulate the conjugation of monoubiquitin $(\mathrm{Ub})$ or polyubiquitin chains to an exposed mitochondrial protein, thereby targeting the organelle to autophagic degradation. p62 and NBR1 are autophagic receptors that bind both Ub and ATG8/LC3 on the phagophore (Ref. 116), which might constitute the mechanistic link between mitochondrial ubiquitylation and mitophagy. The mTOR signalling pathway acts as a major positive modulator of mitochondrial metabolism and biogenesis. We propose that the inhibition of mTORC1 upon mitochondrial damage caused by loss of ATP and consequential activation of AMPK, might be crucial to ensure an enhancement of the autophagic degradation of defective mitochondria. Abbreviations: AMPK, AMPactivated protein kinase; Aup1p, yeast mitochondrial protein phosphatase homologue; BNIP3L/NIX, BCL2/ adenovirus E1B $19 \mathrm{kDa}$ protein-interacting protein 3-like; LC3, light chain 3 protein; LKB1, serine/threonineprotein kinase 11; mTOR, mammalian target of rapamycin; mTORC1, mTOR complex 1; NBR1, next to BRCA1 gene 1 protein; OM, outer membrane; OPA1, optic atrophy 1; Uth1p, yeast outer mitochondrial membrane protein.

Accession information: doi:10.1017/S1462399410001456; Vol. 12; e12; April 2010 (c) Cambridge University Press 2010. Re-use permitted under a Creative Commons Licence - by-nc-sa. 
provided by molecular chaperones and endogenous proteases, which respectively detect and eliminate abnormally folded and aggregated proteins generated by ROS. Oxidative damage to mitochondria is also counteracted at the organelle level by components of the fusion and fission machinery, which ensure the maintenance of a functional population of organelles (Ref. 5). This occurs through the alteration of mitochondrial network dynamics and leads to the segregation of defective mitochondria, which are eventually removed via autophagy (mitophagy). Significantly, a major consequence of mitochondrial damage is loss of ATP, which results in a decrease in the ratio of ATP to AMP. This, in turn, can lead to the activation of a cellular signal-transduction pathway where the AMP-activated protein kinase (AMPK) acts as a master sensor of intracellular energy status (Ref. 6) and reduces the activity of the mammalian target of rapamycin complex 1 (mTORC1). mTORC1 is a major positive modulator of mitochondrial metabolism and biogenesis (reviewed in Ref. 7). It is supposed that an mTORC1-dependent decrease in mitochondrial biogenesis could indirectly promote the autophagic segregation of defective mitochondria by ensuring that mitochondrial dynamics processes are used for the clearance of dysfunctional mitochondria, as opposed to their biogenesis (Fig. 2).

Ultimately, if mitochondrial damage is too severe and overwhelms the cellular networks of protective mechanisms, dysfunctional organelles break open, releasing a series of apoptotic factors that ultimately cause cell death (reviewed in Ref. 8). This removal of cells with severely injured mitochondria has been suggested to be another layer of protection that acts at the level of the organism (Ref. 9). Nevertheless, the demise of postmitotic cells such as neurons can result in irreparable damage to the organism.

The chronic and selective loss of neuronal cells is indeed a typical feature of most neurodegenerative diseases and results in the progressive impairment of function in the central (CNS) and/ or peripheral nervous system (PNS). Although they affect different neuronal cell populations, neurodegenerative diseases share remarkable common traits, e.g., enhanced neuronal cell death, defects in axonal transport and the accumulation of dysfunctional mitochondria.
In addition to their primary role in cellular metabolism, mitochondria are active players in $\mathrm{Ca}^{2+}$ homeostasis. Under conditions of cellular $\mathrm{Ca}^{2+}$ overload, in association with oxidative stress, mitochondrial $\mathrm{Ca}^{2+}$ uptake leads to the collapse of $\Delta \psi_{\mathrm{m}}$, which sensitises cells to apoptosis. In this regard, it is noteworthy that alterations of mitochondrial $\mathrm{Ca}^{2+}$-regulating proteins (Bcl-2 family members and uncoupling proteins) are implicated in age-related neuronal pathologies (Ref. 10).

The high density of mitochondria within neurons could provide a rationale for the sensitivity of the CNS to energy deficits due to mitochondrial dysfunction. Nevertheless, it is still debated whether mitochondrial impairment and oxidative damage are aetiological factors, or are solely the consequences of neurodegeneration. In this review, we provide a synopsis of the current advances in the knowledge of mitochondrial quality-control systems, with particular emphasis on how disturbances in the molecular components of such systems might contribute to neurological diseases.

\section{Mitochondrial dysfunction and Parkinson disease}

Mitochondrial dysfunction has long been associated with the onset of neurodegenerative states, including the selective loss of dopaminergic neurons in Parkinson disease (PD). PD is the most common neurodegenerative movement disorder, and it affects $1 \%$ of the population above the age of 60 . The critical loss of pigmented neurons in the substantia nigra underlies the motor impairment, which is the most common clinical feature of the disease. However, neuronal loss in regions of the brain controlling autonomic functions, cognition and mood is also implicated. Neurons that degenerate in $\mathrm{PD}$ often contain intracellular inclusions of $\alpha$-synuclein (SNCA), which is a major component of the so-called Lewy bodies (Ref. 11).

Despite the fact that the majority of PD cases occur sporadically as a result of unknown causes, reduced levels of mitochondrial complex I activity are generally associated with the disease (Refs 12, 13, 14). Additionally, the toxins rotenone and MPTP cause an acute and irreversible parkinsonian syndrome that might involve the specific inhibition of complex I (Refs 15, 16). This suggests that one of the main

Accession information: doi:10.1017/S1462399410001456; Vol. 12; e12; April 2010 (c) Cambridge University Press 2010. Re-use permitted under a Creative Commons Licence - by-nc-sa. 
causes of neuron loss and motor impairment in $\mathrm{PD}$ is toxin-induced mitochondrial stress in dopaminergic neurons (reviewed in Ref. 17).

Although classically considered to be a nongenetic disorder because of the large number of sporadic cases, genetic analyses of the small proportion of familial PD cases have led to significant insights into its pathogenesis.

Mutations in SNCA ( $\alpha$-synuclein) and LRRK2 (leucine-rich repeat kinase 2) genes mediate autosomal-dominant forms of PD, whereas mutations in PARK2 [Parkinson disease (autosomal recessive, juvenile) 2; parkin], PARK7 [Parkinson disease (autosomal recessive, early onset) 7; DJ-1] and PINK1 (PTEN-induced putative kinase 1) cause the disease in an autosomal-recessive manner. Mutations in HTRA2, which encodes the mitochondrial serine protease (HtrA2 serine peptidase; OMI) have also been described in families affected by PD (see Ref. 18 for a review). However, the importance of HTRA2 mutations in the development of PD has been questioned by independent studies, indicating that the role of this protease in the development of PD is still controversial.

Interestingly, the major common functional effects of the proteins encoded by these genes relate to mitochondria: PINK1 is a kinase regulated by a canonical N-terminal mitochondrial-targeting sequence (Ref. 19); DJ-1 is a molecular chaperone that has been proposed to be localised to the mitochondria upon oxidative stress (Refs 20, 21, 22) and stabilise complex I (Ref. 23); Parkin and PINK1 seem to be involved in the regulation of mitochondrial dynamics, morphology and turnover (Refs 24, 25, 26, 27, 28); and finally HTRA2/OMI and PINK1 have been shown to be components of the same mitochondrial stresssensing pathway (Ref. 29). Given the fact that $\alpha-$ synuclein and LRRK2 are partially localised to mitochondria (Refs 30,31), the majority of genes associated with PD so far implicate, either directly or indirectly, the involvement of mitochondria in the pathogenesis of this disease (Table 1). Molecular dissection of the contribution of these gene products to mitochondrial function is currently underway and is likely to be important for a better understanding of PD (reviewed in Ref. 32).

Any given mitochondrion is not a discrete, autonomous organelle (Ref. 2); in fact, the entire mitochondrial population within a cell is in constant flux driven by a series of fusion and fission events. The dynamic nature of the mitochondrion population not only determines mitochondrial morphology and copy number (Ref. 33), but it also provides additional protection against mitochondrial damage (Fig. 2). Recently, it was proposed that PINK1 and Parkin regulate mitochondrial dynamics through interaction with the fusion-fission machinery (Refs 24, 25, 26). However, it is also conceivable that these proteins affect mitochondrial dynamics indirectly by perturbing calcium and ROS homeostasis (Refs 27, 34). Several groups reported that loss of Pink1 or parkin in Drosophila leads to significant mitochondrial enlargement, suggesting a defect in mitochondrial fission (Refs 35, 36). Interestingly, the phenotypes of Pink1 or Parkin mutant flies are very similar, and although Pink1 is unable to rescue the defects caused by the lack of parkin, overexpression of parkin rescues the mitochondrial pathology induced by the loss of Pink1. By contrast, PINK1deficient mammalian cells show fragmented and truncated mitochondria; nevertheless, the molecular interaction between Parkin and PINK1 is conserved in mammalian cells (Refs $37,38,39$ ).

Parkin acts as an E3 ubiquitin ligase, and it is mostly localised to the cytosol. However, it has been shown in vitro that Parkin is recruited to the mitochondria upon loss of $\Delta \psi_{\mathrm{m}}$, leading to a selective autophagic engulfment and elimination of dysfunctional organelles (Ref. 28) (Fig. 3). According to Chung and colleagues, this recruitment is dependent on the direct phosphorylation of Parkin on Thr175 by PINK1 (Ref. 40), whose kinase domain faces the cytosol (Ref. 41). Considering that downregulation of PINK1 causes loss of mitochondrial membrane potential in several mammalian systems (Refs 19, $37,42)$, it seems plausible to propose a role for PINK1 in flagging dysfunctional mitochondria for degradation. The mitochondrial pathology in PINK1-deficient animals might be caused by an insufficient recruitment of Parkin followed by an impairment of mitochondrial turnover (Ref. 43). However, given that Parkin is capable of rescuing mitochondrial dysfunction caused by the loss of PINK1, it is unclear whether a direct molecular interaction between PINK1 and Parkin is essential for efficient removal of damaged mitochondria.

PINK1 and Parkin seem to share the same genetic pathway, which probably involves the mitochondrial quality-control machinery.

Accession information: doi:10.1017/S1462399410001456; Vol. 12; e12; April 2010 (C) Cambridge University Press 2010. Re-use permitted under a Creative Commons Licence - by-nc-sa. 


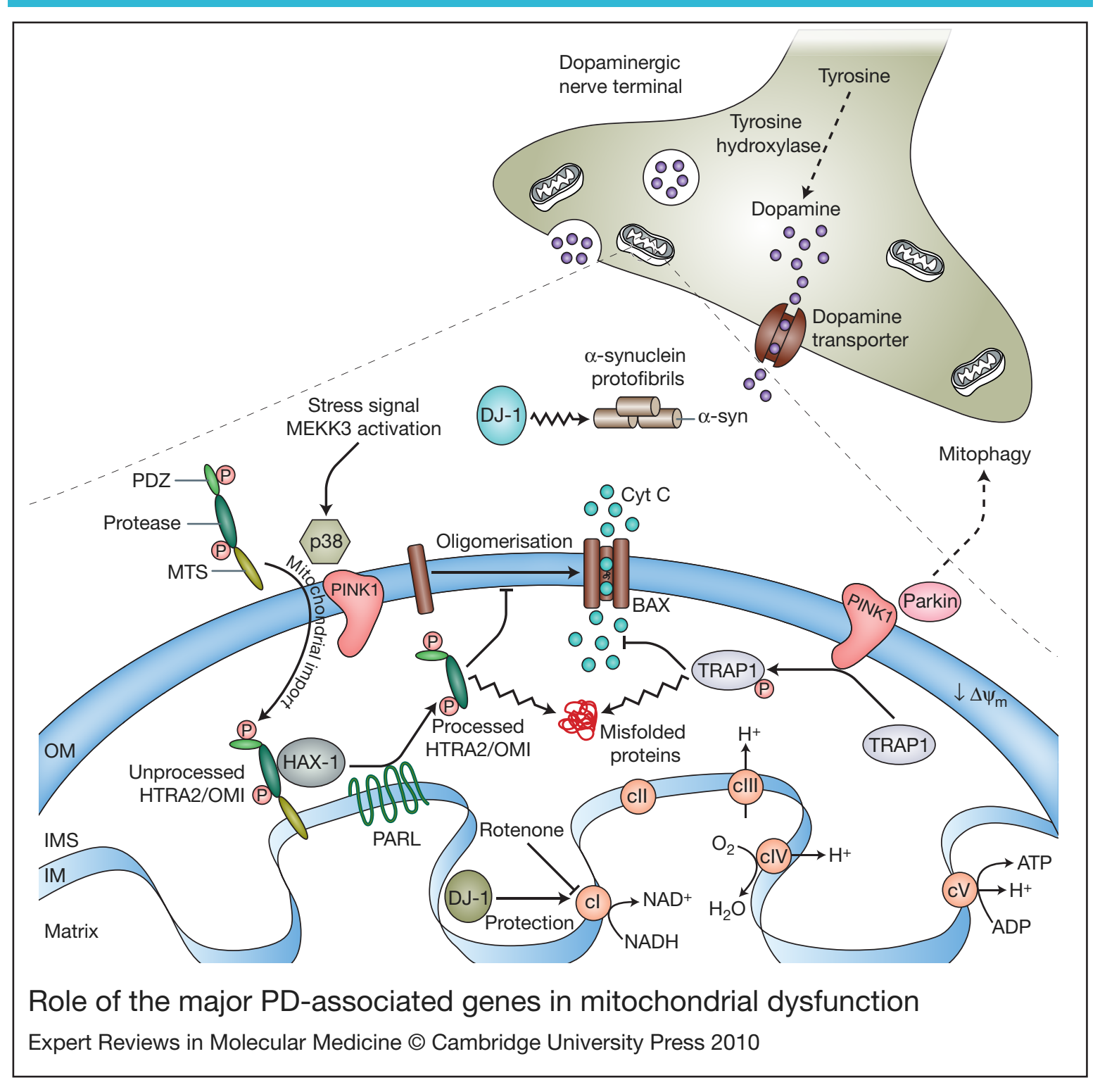

Figure 3. Role of the major PD-associated genes in mitochondrial dysfunction. (See next page for legend.)

Additionally, two intermembrane space (IMS) proteins involved in mitochondrial quality control are substrates of PINK1: the chaperone TRAP1/ Hsp75 (Ref. 44) and the serine protease HTRA2/OMI (Ref. 29). Under stress conditions, these proteins interact with PINK1 (Fig. 3) and are subsequently activated to prevent the accumulation of misfolded proteins. Given the proposed role of toxins such as rotenone and MPTP in acute parkinsonian syndrome, it is attractive to argue that defects in proteins involved in mitochondrial-stress pathways could increase the susceptibility towards neurodegeneration in individuals exposed to environmental toxins.

In addition to mitochondrial dysfunction, protein aggregation also has a major role in PD pathogenesis. However, it remains to be determined whether there is a convergence between mitochondrial dysfunction and protein aggregation. $\alpha$-synuclein is a fibrillar aggregationprone protein that is a main component of Lewy bodies and is believed to contribute to PD by toxic gain-of-function effects. Although this protein is mostly cytosolic, mitochondrial abnormalities were observed in several transgenic

Accession information: doi:10.1017/S1462399410001456; Vol. 12; e12; April 2010 (c) Cambridge University Press 2010. Re-use permitted under a Creative Commons Licence - by-nc-sa. 
Figure 3. Role of the major PD-associated genes in mitochondrial dysfunction. (See previous page for figure.) Many genes associated with Parkinson disease (PD) implicate a role for mitochondria in the pathogenesis of the disease. The serine protease HTRA2-OMI is synthesised as an inactive precursor containing a mitochondrial-targeting sequence (MTS). In response to the activation of the MEKK3-p38 stress-kinase-signalling pathway, HTRA2/OMI is phosphorylated by p38 in a PINK1-dependent manner and imported into the intermembrane space (IMS), where it has been reported to bind to the Bcl-2-family-related protein Hax-1 (Ref. 117). Despite the fact that this interaction is controversial (Ref. 118), it has been suggested to promote the proteolytic processing of HTRA2/OMI by the mitochondrial protease PARL. Active HTRA2/OMI is thought to be involved in the degradation of misfolded proteins present in the IMS (Ref. 119) and to prevent the oligomerisation of the activated form of BAX on the outer membrane (OM), thus avoiding apoptosis (Ref. 117). Under oxidative stress conditions, PINK1 also interacts with the mitochondrial molecular chaperone TRAP1. Once phosphorylated, TRAP1 inhibits oxidative-stress-induced cytochrome C (Cyt C) release, prevents misfolding and promotes the correct assembly of mitochondrial proteins. DJ-1 is a cytosolic oxidative-stress-regulated chaperone, which redistributes from the cytosol to the mitochondria upon oxidation of specific cysteine residues. In the cytoplasm, DJ-1 prevents the aggregation and toxicity of $\alpha$-synuclein ( $\alpha$-syn); in mitochondria, it has been suggested to protect respiratory complex I (cl) from oxidative-stress-mediated inactivation (Ref. 23). Another protein involved in the mitochondrial stress response is Parkin, a cytosolic E3-ubiquitin ligase, which is selectively recruited to uncoupled or dysfunctional mitochondria, targeting them for autophagy (Ref. 28). A model is illustrated whereby PINK1, acting upstream of Parkin, might regulate mitochondrial function by sensing mitochondrial damage, recruiting Parkin and inducing mitophagy. Abbreviations: ADP, adenosine diphosphate; ATP, adenosine-5'triphosphate; cll, complex II; clll, complex III; cIV, complex IV, cV, complex V; HAX-1, HCLS1-associated protein X-1; HTRA2, high-temperature requirement A2; IM, mitochondrial inner membrane; MEKK3, mitogen-activated protein kinase kinase kinase 3; PINK1, PTEN-induced putative kinase 1; PD, Parkinson disease; PDZ domain, post-synaptic density protein (PSD95), Drosophila discs large tumour suppressor (DlgA) and zonula occludens-1 protein (zo-1) domain; TRAP1, TNF-receptor associated protein; $\Delta \psi_{\mathrm{m}}$, mitochondrial membrane potential.

mouse models that either lack or overexpress wildtype or mutant $\alpha$-synuclein (Refs 45,46 ), suggesting that it has a physiological role in the mitochondria (Ref.47), where it might interact with mitochondrial proteins. Recent work has shown that loss of PINK1 leads to an increase of $\alpha$-synuclein aggregation (Ref. 48), whereas overexpression of PINK1 was able to suppress $\alpha$-synuclein-induced phenotypes in Drosophila (Ref. 49). Moreover, the chaperone DJ-1 has been shown to redistribute to mitochondria upon oxidative stress (Refs 20, $21,22)$ and to prevent the aggregation and toxicity of $\alpha$-synuclein in an oxidation-dependent manner (Refs 41, 50, 51).

Whether or not DJ-1 recruitment is regulated in the same manner as Parkin remains to be determined. However, it has already been demonstrated that Parkin, PINK1 and DJ-1 form a functional ubiquitin E3 ligase complex that promotes ubiquitylation and degradation of unfolded or misfolded proteins (Ref. 52). This complex might thus be the missing link between mitochondrial function and protein aggregation.

Finally, PINK1, DJ-1 and Parkin seem to interact in vivo with LRRK2, another PD-associated protein (Refs 53, 54). LRRK2 is a serine/threonine kinase responsible for the phosphorylation of actin crosslinkers, moesin (MSN), ezin (EZR) and radixin (RDX) (Ref. 55), as well as the eukaryotic initiation factor $4 \mathrm{E}$ (eIF4E)-binding protein (4E-BP), which is a negative regulator of eIF4E-mediated protein translation and a key mediator of various stress responses. 4E-BP activity is also regulated by the TOR signalling pathway. Activated TOR hyperphosphorylates 4E$\mathrm{BP}$, inhibiting it and promoting 5'-cap-dependent translation. A switch from cap-dependent to capindependent initiation of translation seems to be a key event in mediating the survival response to various physiological stresses (Ref. 56). In support of this, Whitworth and colleagues recently showed that loss of Drosophila Lrrk2 as well as treatment with rapamycin, an inhibitor of TOR, lead to the activation of $4 \mathrm{E}-\mathrm{BP}$, and this suppresses Pink1 and parkin mutant phenotypes in flies. Taken together, and with the caveat that some of these observations await confirmation, it is attractive to suggest that LRRK2 might have a role in the regulation of mitochondrial homeostasis by controlling signalling pathways involved in protein translation.

As described here, the most recent work has emphasised the central importance of mitochondrial dysfunction in PD. Nevertheless,

Accession information: doi:10.1017/S1462399410001456; Vol. 12; e12; April 2010 (C) Cambridge University Press 2010. Re-use permitted under a Creative Commons Licence - by-nc-sa. 
we still need to consider several questions: is this mitochondrial pathway also relevant to sporadic forms of PD? How can we exploit our knowledge of mitochondrial involvement in PD to develop better therapies? We believe that new insights from future studies will provide us with neuroprotective therapies that could make a difference in the treatment of PD.

\section{Mitochondrial dysfunction in Alzheimer disease}

Alzheimer disease (AD) is a progressive and irreversible pathology that affects millions of people worldwide, and it represents the most common form of dementia among elderly people. $\mathrm{AD}$ has been attracting increasing attention from both the scientific community and governments because of its increasing social impact and costs. By the year 2050, 50\% of people 85 years and older will be afflicted with $\mathrm{AD}$ (Ref. 57), making this disease a health priority.

AD can be classified into sporadic AD and familial AD (FAD). Only a small fraction (approximately $10 \%$ ) of AD cases are familial (Ref. 58), and show autosomal-dominant transmission within affected families. Sporadic AD accounts for the majority of cases, with ageing being the most relevant known risk factor. In addition to age and family history, an increased risk of developing the disorder both early and late-onset AD - is associated with apoEs4 (Ref. 59), one of three allelic variants of the human $A P O E$ gene. More recently, variants of clusterin (CLU) and phosphatidylinositol-binding clathrin assembly protein (PICALM) proteins have also been associated with AD (Ref. 60). A characteristic feature of all forms of $\mathrm{AD}$ is progressive neuronal cell death in brain regions displaying high plasticity, which is caused by the accumulation of intraneuronal neurofibrillary tangles (NFTs) and extracellular $\beta$-amyloid plaques. NFTs consist of abnormally phosphorylated tau protein, which is polymerised into paired helical filaments (PHFs); amyloid plaques are composed of $\beta$-amyloid peptide $(\mathrm{A} \beta 1-42)$, which polymerises into insoluble fibrils with high $\beta$-sheet content.

The $A \beta$-aggregation process has been considered for years to be the most relevant phenomenon implicated in the aetiology of AD. However, mounting evidence is now highlighting the role of mitochondrial impairment in the pathology of $\mathrm{AD}$, and the two events are reciprocally related. In particular, the finding that $A \beta$ species accumulate intracellularly in the mitochondria (Ref. 61), and the fact that this occurs before their extracellular accumulation, has changed the focus of potential therapies against this pathology. These findings provide a direct link between $A \beta$ and the pathological dysfunctions of the mitochondria found in $\mathrm{AD}$, such as deficits in tricarboxylic acid (TCA) cycle and/or complex IV. Although the molecular origin of the intramitochondrial $A \beta$ is still unknown, the in vivo and in vitro consequences of these deposits in the cell have, in part, been elucidated. The presence of $\beta$-amyloid aggregates in the organelle has been shown to impair the enzymatic activity of cytochrome c oxidase and inhibit the activity of mitochondrial amyloid- $\beta$ binding alcohol dehydrogenase (ABAD) (Fig. 4). The selective decrease in complex IV observed in the brains of AD patients (Refs 62,63) could be responsible for an imbalance in the electron transport chain (ETC) energy-extracting mechanism, which, in turn, would compromise the general metabolism of the neurons.

The process of $A \beta$ aggregation is accompanied by the generation of ROS and lipid peroxidation. At the mitochondrial level, such neurotoxic actions affect the components of the OXPHOS by depletion of ATP levels (Ref. 64), and they exert a secondary effect on the mitochondrial ATPase. This, in turn, results in the altered regulation of the calcium elevations in which the ATPase is implicitly involved (Ref. 65). Furthermore, the toxicity of A $\beta$ peptides which is associated with their oligomeric state, rather than their fibrillar insoluble state (see Ref. 66 for a review) - causes a rapid increase of intracellular calcium that eventually leads to cell death. A pivotal role in cell death commitment is played by the opening of the mitochondrial permeability transition pore (mPTP), which determines the collapse of $\Delta \psi_{\mathrm{m}}$ and the subsequent release of IMS proapoptotic factors. The molecular basis for this pathogenic mechanism was provided by the work of $\mathrm{Du}$ and colleagues (Ref. 67) in both transgenic mouse models and human AD brains, where the $A \beta$ peptide has been shown to interact with cyclophilin D (CYPD; CypD), a mitochondrial matrix protein involved in $\mathrm{MPTP}$ formation (Fig. 4).

Several cell models document the accumulation of the full-length amyloid precursor protein (APP) in the outer mitochondrial membrane as

Accession information: doi:10.1017/S1462399410001456; Vol. 12; e12; April 2010 (C) Cambridge University Press 2010. Re-use permitted under a Creative Commons Licence - by-nc-sa. 
Figure 4. Mitochondrial dysfunction in AD. Degenerating neurons in brain areas affected by Alzheimer disease (AD) (e.g. hippocampus and cerebral cortex) present typical intracellular neurofibrillary tangles (NFTs) and extracellular accumulation of $\beta$-amyloid plaques. $A \beta$ is produced from the metabolism of amyloid precursor protein (APP) through sequential cleavage by $\beta$ - and $\gamma$-secretases, and it is prone to form toxic oligomeric structures both inside and outside the cell. APP and A $\beta$ can affect mitochondrial function by different mechanisms. APP is targeted to mitochondria, where it forms complexes with the translocases of the outer and inner membranes (TOM and TIM), which drive the import of mitochondrial proteins in cooperation with molecular chaperones. However, the presence of an acidic domain within APP might be responsible for preventing its translocation into mitochondria. As a consequence, the import of mitochondrial proteins, such as respiratory chain subunits, is reduced, and this event is associated with increased free radical generation and reduced activity of the electrontransport chain. Other aspects of amyloid metabolism involve mitochondria: intramitochondrial $A \beta$ has been shown to interact with amyloid- $\beta$-binding alcohol dehydrogenase (ABAD) and to produce reactive oxygen species (ROS). A $\beta$ also interacts with cytochrome $c$ oxidase, thus decreasing the activity of complex IV (cIV). Furthermore, presequence peptidase PreP and HTRA2/OMI serine protease have been shown to degrade A $\beta$ oligomers, thus providing a mechanism to detoxify this metabolite. Intramitochondrial $A \beta$ directly interacts with cyclophilin $D$ (CypD), a component of the mitochondrial permeability transition pore (mPTP), which is located in the mitochondrial matrix. This interaction makes the channel more sensitive to $\mathrm{Ca}^{2+}$ and stimulates $\mathrm{mPTP}$ opening, thus raising the permeability of the mitochondrial inner membrane (IM) and eventually disrupting the mitochondrial outer membrane (OM). As a result, deregulation of the mPTP opening determines a functional disorder that triggers cell death. Abbreviations: AD, Alzheimer disease; ATP, adenosine-5'-triphosphate; cl, complex I; cll, complex II; clll, complex III; cV, complex V; HTRA2, HtrA2 serine peptidase; $\Delta \psi_{\mathrm{m}}$, mitochondrial membrane potential.

Accession information: doi:10.1017/S1462399410001456; Vol. 12; e12; April 2010 (C) Cambridge University Press 2010. Re-use permitted under a Creative Commons Licence - by-nc-sa. 
another molecular event that is responsible for mitochondrial dysfunction, owing to its translocational arrest (Ref. 68). Although several aspects of the pathogenesis of $\mathrm{AD}$ remain unsolved, the turnover and degradation of APP and $A \beta$ in the mitochondrial compartment appear to be two fundamental processes whose dysregulation might lead to dramatic consequences on the functioning of the organelle, and ultimately, of the cell as a whole.

In transgenic mice, elimination of the aberrant forms of these proteins can reverse the neurological deficits without affecting the number of neurons (Refs 69, 70, 71). From a therapeutic perspective, these findings suggest that some neurological defects that are associated with $\mathrm{AD}$ might be caused by neuronal dysfunction rather than the loss of neurons. Drugs aimed at enhancing the removal of abnormal $A \beta$ assemblies might help to inhibit their toxic action on mitochondria and, by extension, neurons. At present, several studies are attempting to identify in vitro and in vivo modulators that influence $A \beta$ aggregation, as well as tau in the NFTs. A potential role for HTRA2/OMI protease in the clearance of $\mathrm{A} \beta$ oligomers from within the mitochondria has been recently indicated (Ref. 72). A similar function in degradation of mitochondrial $A \beta$ has been suggested for the mitochondrial matrix metallopeptidase PreP (prolyl endopeptidase, PREP), an organellar functional analogue of the human insulindegrading enzyme (IDE) (Ref. 73) (Fig. 4).

The mitochondrial dysfunction found in $\mathrm{AD}$ has also been related to mechanisms that are independent of $A \beta$. The majority of hereditary $\mathrm{AD}$ cases (autosomal dominant) carry mutations in the presenilin genes PSEN1 and PSEN2, the subcomponent of $\gamma$-secretase that is responsible for APP cleavage to produce $A \beta$ peptide. Specifically, mutations in PSEN1 have been shown to destabilise mitochondrial functions through two possible mechanisms by: (1) perturbing endoplasmic reticulum (ER) calcium handling, which in turn promotes excessive mitochondrial $\mathrm{Ca}^{2+}$ uptake and apoptosis (Ref. 74); and (2) impairing the axonal transport of the organelle, which affects the normal synaptic activity of neurons (Ref. 75).

Altogether, these studies have attributed the causes of $\mathrm{AD}$ to two inter-related pathogenic events: aggregation of misfolded proteins, and mitochondrial dysfunction; however, their interrelationship needs further investigation. Despite the broad agreement that both processes are likely to have pivotal roles in AD progression, there is insuficient evidence supporting the notion that mitochondrial alterations are a primary cause of this disease. Instead, we favour the notion that mitochondrial dysfunction is a secondary event in the disease process, which nevertheless might be a key determinant of neurodegeneration in AD.

Although mitochondrial dysfunction and aberrant protein degradation are clearly related to $\mathrm{AD}$, many questions remain unanswered. A major problem inherent to AD is the absence of early diagnostic tests, which is due in part to the lack of reliable predisease biomarkers. Several new approaches designed to target the formation of $\beta$-amyloid aggregates are under investigation in clinical trials, with the aim of slowing or halting the progression of $\mathrm{AD}$ (refer to the Alzheimer's association page in the Further Reading section below). However, synapse loss in the neocortex and hippocampus is a well-documented structural feature of the brain lesion in $\mathrm{AD}$, and it is reported to be an early event in $\mathrm{AD}$ (Refs 76, 77). For that reason, it would be useful to look for markers of synaptic loss, such as synaptophysin (Refs 78, 79). Future studies should also investigate the molecular aspects of mitochondria in neurons of animal models of AD. It would be interesting to study the effects of specific manipulations of mitochondrial functions in these cells, to determine whether the mitochondrial damage in $\mathrm{AD}$ can be reversed by stimulating autophagy and/or mitochondrial biogenesis.

\section{Huntington disease and mitochondrial dysfunction}

Huntington's disease (HD) is a fatal, dominantly inherited neurodegenerative disorder characterised by chorea, involuntary movements, and cognitive impairments. Symptoms result from the selective loss of long-projection neurons known as medium spiny neurons. These neuronal cells release $\gamma$-aminobutyric acid (GABA) in the striatal brain regions that control movement, memory and emotions. HD is slowly progressive, and patients survive for about 15-20 years from disease onset (for reviews, see Refs 80,

Accession information: doi:10.1017/S1462399410001456; Vol. 12; e12; April 2010 (c) Cambridge University Press 2010. Re-use permitted under a Creative Commons Licence - by-nc-sa. 
11). Tremendous progress has been made since the discovery of the HTT gene in 1993. HDis caused by a genetic mutation that results in an expanded polyglutamine-encoding repeat within exon 1 of the HTT gene (Ref. 81).

The HTT gene product, Huntingtin (HTT, Htt), is an extremely large protein of $350 \mathrm{kDa}$, which is ubiquitously expressed in the brain and peripheral tissues. It has been reported to act as a scaffold protein that regulates signalling pathways, and vesicle and organelle trafficking. Although Htt is mostly cytoplasmic, it is also found at lower concentrations in multiple subcellular compartments, such as the plasma membrane, nucleus, endoplasmic reticulum, Golgi and mitochondria.

A polyglutamine stretch of less than 35 in the Nterminus of $\mathrm{Htt}$ is normal and does not cause disease. However, an abnormal stretch of 36-39 glutamine residues results in incomplete penetrance of the disease, and 40 or more results in HD with full penetrance (see Ref. 82 for a review).

Strong evidence suggests that mitochondrial impairment has a key role in HD pathogenesis (Ref. 83). Mutant Htt (mtHTT, mtHtt) might cause its neurotoxicity by evoking defects in mitochondria, which in turn lead to a bioenergetic failure, HD-linked neuronal dysfunction and cell death. Indeed, postmortem brain samples of HD patients exhibit reduced activity of mitochondrial respiratory complexes II, III and IV (Ref. 84); and humans exposed to 3-nitropropionic acid (3-NP), a selective inhibitor of succinate dehydrogenase and complex II, exhibit motor dysfunction similar to that observed in HD patients (Ref. 85).

Moreover, several harmful effects of mtHtt on mitochondria have been reported, including: reduced ATP levels in synaptic terminals (Ref. 86); mitochondria depolarisation at lower $\mathrm{Ca}^{2+}$ loads (Ref. 87); increased sensitivity to $\mathrm{Ca}^{2+}$ overload and N-methyl-D-aspartic acid (NMDA) receptor-mediated neuronal apoptosis (Ref. 88); and a reduced threshold for mPTP opening and cytochrome c release (Ref. 89). Although the bioenergetic deficits in HD are well known, mtHtt can also adversely affect mitochondria by modifying gene transcription. For instance, mtHtt binds to the tumour suppressor p53 and increases its levels, which, in turn, results in transcriptional activation of its proapoptotic mitochondrial targets Bax and
Puma, causing mitochondrial damage. Since the loss of p53 prevents mtHtt-mediated neurodegeneration in Drosophila (Ref. 90), we can easily assume that altered p53 transcriptional activity results in mitochondrial dysfunction and neuronal loss.

PPARGC1A [PGC- $1 \alpha$, peroxisome proliferatoractivated receptor (PPAR)- $\gamma$ coactivator $1 \alpha$ ] is another gene whose expression seems to be regulated by $\mathrm{mtHtt}$. By interacting with the promoter and interfering with CREB-dependent PPARGC1A gene expression, $\mathrm{mtHtt}$ represses the transcription of this gene (Ref. 91). PGC- $1 \alpha$ is a nuclear co-activator that has a major role in mitochondrial biogenesis; therefore, inhibition of PGC- $1 \alpha$ expression limits the ability of the vulnerable neurons to adequately respond to energy demands in HD.

Although the transcriptional deregulation of $\mathrm{mtHtt}$ is of great relevance, it cannot fully explain all the mitochondrial defects observed in HD. Recent studies indicate that impaired mitochondrial trafficking along axons and dendrites might also have an important role in the disease pathology (see Ref. 30 for a review). Mitochondria are dynamically transported along lengthy neuronal processes to provide energy to nerve terminals and maintain the normal neuronal function. ATP-dependent motor proteins regulate such mitochondrial movement: kinesins mediate anterograde transport (away from the cell body), and dynein-dynactin regulates retrograde transport (toward the cell body) (Ref. 92). Wild-type Htt seems to regulate the trafficking of endocytic vesicles by binding to Htt-associated protein 1 (HAP1) (see Ref. 30 for a review). This protein complex might act as a docking platform that interacts with the molecular motor dyneindynactin and kinesin, and it is known to regulate microtubule-mediated BDNF (brainderived neurotrophic factor) vesicle and mitochondrial transport (Refs 93, 94). Furthermore, phosphorylation of Htt seems to act as a molecular switch for bidirectional transport in neurons (Ref. 95) (Fig. 5).

Whereas wild-type Htt promotes axonal BDNF vesicle trafficking, $\mathrm{mtHtt}$ disrupts the formation of trafficking complexes and impairs vesicle transport (Ref. 93). Abnormal interaction of $\mathrm{mtHtt}$ with motor proteins seems to be the main cause of the trafficking defects; however,

Accession information: doi:10.1017/S1462399410001456; Vol. 12; e12; April 2010 (c) Cambridge University Press 2010. Re-use permitted under a Creative Commons Licence - by-nc-sa. 
Figure 5. Role of Htt protein in mitochondrial trafficking and dynamics. Mitochondrial movement in neurons is highly diverse and complex. Normal $\mathrm{Htt}$ protein regulates anterograde (away from the cell body) and retrograde (towards the cell body) transport of mitochondria by interacting with several trafficking mediators. Htt stimulates trafficking by binding to HAP1, which in turn, leads to interaction with the motor proteins dynein-dynactin and kinesin. Phosphorylation of $\mathrm{Htt}$ acts as a molecular switch for anterograde versus retrograde mitochondrial transport. When $\mathrm{Htt}$ is phosphorylated, kinesin-1 is recruited and promotes anterograde transport; conversely, when $\mathrm{Htt}$ is unphosphorylated, kinesin-1 detaches from the motor complex and induces a switch to retrograde transport (Ref. 95). In addition to migration and movement, mitochondria undergo cycles of fusion and fission. The key mitochondrial fission regulator is dynamin-related protein 1 (DRP1). Similarly to dynamin, DRP1 seems to act as a mechano-enzyme to constrict and divide mitochondria. Given that $\mathrm{Htt}$ interacts with dynamin, one can speculate that $\mathrm{Htt}$ might regulate fission by interacting with DRP1. Abbreviations: HAP1, Htt-associated protein 1; Htt, Huntingtin protein; P, phosphate.

mtHtt aggregates might also contribute by acting as a physical roadblock (Refs 2, 86), or by sequestering wild-type Htt and components of the trafficking machinery (Ref. 96).

Neuronal function is particularly dependent on the intracellular trafficking of organelles and molecules. Defective mitochondrial transport ultimately impairs neuronal transmission and results in synaptic damage and selective neuronal loss. In addition to blocking mitochondrial movement, it has been reported that $\mathrm{mtHtt}$ induces an imbalance in mitochondrial fission and fusion (Fig. 2). According to Monteiro and colleagues (Ref. 97),

Accession information: doi:10.1017/S1462399410001456; Vol. 12; e12; April 2010 (c) Cambridge University Press 2010. Re-use permitted under a Creative Commons Licence - by-nc-sa. 
Table 1. Genes involved in major mitochondria-associated neurodegenerative diseases

\begin{tabular}{|c|c|c|c|c|c|}
\hline Gene & Disease & $\begin{array}{l}\text { Chromosome } \\
\text { location }\end{array}$ & $\begin{array}{l}\text { Subcellular } \\
\text { localisation } \\
\text { of protein }\end{array}$ & $\begin{array}{l}\text { Molecular } \\
\text { function }\end{array}$ & Ref. \\
\hline PINK1 & PD & $1 p 36.12$ & $\begin{array}{l}\text { Mitochondria } \\
(\mathrm{OM})\end{array}$ & $\begin{array}{l}\text { Serine/ } \\
\text { threonine-protein } \\
\text { kinase }\end{array}$ & $120,19,44$ \\
\hline PARK2 & PD & 6q25.2-q27 & Cytosol & $\begin{array}{l}\text { E3 protein- } \\
\text { ubiquitin ligase }\end{array}$ & 28 \\
\hline PARK7 & PD & 1p36.33-p36.12 & Cytosol & $\begin{array}{l}\text { Molecular redox- } \\
\text { sensitive } \\
\text { chaperone }\end{array}$ & 50,51 \\
\hline SNCA & PD & $3 q 22$ & Cytosol & Unknown & \\
\hline LRRK2 & PD & $12 q 12$ & $\begin{array}{l}\text { Mitochondria } \\
\text { (OM) }\end{array}$ & $\begin{array}{l}\text { Serine/threonine } \\
\text { protein kinase }\end{array}$ & 53,54 \\
\hline TRAP1 & PD & $16 p 13.3$ & $\begin{array}{l}\text { Mitochondria } \\
\text { (IMS) }\end{array}$ & $\begin{array}{l}\text { Molecular } \\
\text { chaperone }\end{array}$ & 44 \\
\hline HTRA2 & PD, AD & $2 \mathrm{p} 13.1$ & $\begin{array}{l}\text { Cytosol, } \\
\text { mitochondria } \\
\text { (IMS and } \\
\text { matrix) }\end{array}$ & Serine protease & 29,72 \\
\hline OPA1 & PD & $3 q 28-q 29$ & $\begin{array}{l}\text { Mitochondria } \\
\text { (IMS) }\end{array}$ & $\begin{array}{l}\text { Dynamin-related } \\
\text { GTPase required } \\
\text { for mitochondrial } \\
\text { fusion }\end{array}$ & 24,25 \\
\hline$P A R L$ & PD & $3 q 27.3$ & $\begin{array}{l}\text { Mitochondria } \\
\text { (IM) }\end{array}$ & $\begin{array}{l}\text { Mitochondrial } \\
\text { intramembrane- } \\
\text { cleaving } \\
\text { protease }\end{array}$ & 117 \\
\hline$A P P$ & $A D$ & 21q21.2|21q21.3 & $\begin{array}{l}\text { Plasma } \\
\text { membrane; } \\
\text { mitochondria } \\
(\mathrm{OM})\end{array}$ & $\begin{array}{l}\text { Precursor } \\
\text { molecule for } \\
\text { amyloid beta }(A \beta) \\
\text { generation; } \\
\text { regulator of } \\
\text { synapse } \\
\text { formation and } \\
\text { neural plasticity }\end{array}$ & $61,64,69,70$ \\
\hline PPID & $A D$ & $4 q 31.3$ & $\begin{array}{l}\text { Mitochondria } \\
\text { (matrix space) }\end{array}$ & $\begin{array}{l}\text { Molecular } \\
\text { chaperone; } \\
\text { component of } \\
\text { the mitochondrial } \\
\text { permeability } \\
\text { transition pore }\end{array}$ & 67 \\
\hline PREP & $A D$ & $6 q 22$ & $\begin{array}{l}\text { Mitochondria } \\
\text { (matrix space) }\end{array}$ & $\begin{array}{l}\text { Presequence } \\
\text { protease }\end{array}$ & 73 \\
\hline
\end{tabular}


Table 1. Genes involved in major mitochondria-associated neurodegenerative diseases (continued)

\begin{tabular}{|c|c|c|c|c|c|}
\hline Gene & Disease & $\begin{array}{l}\text { Chromosome } \\
\text { location }\end{array}$ & $\begin{array}{l}\text { Subcellular } \\
\text { localisation } \\
\text { of protein }\end{array}$ & $\begin{array}{l}\text { Molecular } \\
\text { function }\end{array}$ & Ref. \\
\hline HTT & HD & $4 p 16.3$ & $\begin{array}{l}\text { Cytosol, } \\
\text { plasma } \\
\text { membrane, } \\
\text { nucleus, ER, } \\
\text { Golgi, } \\
\text { mitochondria }\end{array}$ & $\begin{array}{l}\text { Scaffold protein } \\
\text { regulating vesicle } \\
\text { and organelle } \\
\text { trafficking }\end{array}$ & 96 \\
\hline PPARGC1A & HD & $4 p 15.1$ & Nucleus & $\begin{array}{l}\text { Transcriptional } \\
\text { coactivator }\end{array}$ & 91 \\
\hline DNM1L & HD & $12 \mathrm{p} 11.21$ & $\begin{array}{l}\text { Cytosol, } \\
\text { mitochondrial } \\
\text { surface }\end{array}$ & $\begin{array}{l}\text { Mitochondrial } \\
\text { GTPase required } \\
\text { for mitochondrial } \\
\text { fission }\end{array}$ & 98 \\
\hline \multicolumn{6}{|c|}{$\begin{array}{l}\text { Abbreviations: AD, Alzheimer's disease; DNM1L, dynamin-1 like protein (DRP1); HD, Huntington disease; } \\
\text { HTRA2, HtrA2 serine peptidase (OMI); HTT Huntingtin (Htt); IM, inner membrane; IMS, Inner membrane space; } \\
\text { LRRK2, leucine-rich repeat kinase 2; OM, outer membrane; OPA1, optic atrophy protein 1, autosomal dominant; } \\
\text { PARK2, Parkinson disease autosomal recessive, juvenile } 2 \text { (parkin); PARK7, Parkinson disease autosomal } \\
\text { recessive, early onset } 7 \text { (DJ-1); PARL, presenilin-associated rhomboid-like protein; PD, Parkinson's disease; } \\
\text { PINK1, PTEN-induced putative kinase 1; PPARGC1A, peroxisome proliferator-activated receptor gamma, } \\
\text { coactivator } 1 \text { alpha (PGC-1); PPID, peptidylprolyl isomerase D (cyclophilin D; CYPD); PREP, prolyl } \\
\text { endopeptidase (PreP); SNCA, } \alpha \text {-synuclein; TRAP1, TNF-receptor-associated protein } 1 \text { (Hsp75). }\end{array}$} \\
\hline
\end{tabular}

the increase in cytotoxicity induced by $\mathrm{mtHtt}$ is probably mediated by an alteration in mitochondrial dynamics, which results in increased mitochondrial fragmentation. Given that $\mathrm{mtHtt}$ interacts with dynamin (Ref. 98) and its distribution pattern in the mitochondria has striking similarities to DRP1 (Ref. 87), it is possible that DRP1 could also form a complex with Htt. Thus, one can hypothesise that normal Htt might regulate fission events by interacting with DRP1, whereas $\mathrm{mtHtt}$ might alter the assembly and function of these complexes, which ultimately leads to imbalances in mitochondrial dynamics. Naturally, further investigation is needed to test this idea.

As described earlier in this review, mitochondrial fragmentation is a common stress response that allows the segregation and elimination of dysfunctional mitochondria by autophagy. Interestingly, autophagy is a major degradation route for $\mathrm{mtHtt}$, and the pharmacological induction of autophagy seems to be of therapeutic value to neurodegenerative disease caused by aggregate-prone proteins such as HD (Refs 85, 99). Based on this, one might speculate that the induction of autophagy is responsible not only for the clearance of the mutant protein, but also for removal of the dysfunctional mitochondria present in the HD-associated neurons.

\section{Clinical implications}

Ageing is the most important risk factor for common neurodegenerative disorders. In the CNS, the physiological process of ageing has been associated with an elevated mutation load in mtDNA, defects in mitochondrial respiration and increased oxidative damage. Indeed, this mitochondrial loss of function seems to be a consequence of the cellular deterioration that occurs with age, which compromises mitochondrial biogenesis and turnover. Interestingly, it has been suggested that induction of mitochondrial biogenesis through pharmacological (bezafibrate) or metabolic modulation of the PPAR-PGC- $1 \alpha$ pathway could represent an effective therapeutical approach for mitochondrial disorders (Ref. 100).

Accession information: doi:10.1017/S1462399410001456; Vol. 12; e12; April 2010 (c) Cambridge University Press 2010. Re-use permitted under a Creative Commons Licence - by-nc-sa. 
Therapeutic options currently available for patients affected by neurodegenerative diseases are extremely limited. Although characterised by specific attributes and pathological hallmarks, most neurodegenerative diseases have features of mitochondrial dysfunction, which converge in metabolic alterations that ultimately lead to neuronal cell death and affect the brain physiology. Consequently, possible therapies could be aimed at restoring the normal function of these organelles. With regards to $\mathrm{AD}$, recent data suggest that cognitive decline is correlated with selective abnormalities in TCA cycle enzymes of mitochondria (Ref. 101). So far, treatments designed to overcome these defects, by administration of glucose and insulin, have improved memory in AD patients (Ref. 102); however, the benefits are transient. Interesting new data are emerging for long-term benefits to AD patients from studies on Dimebon, an antihistamine drug. In contrast to the conventional drugs for AD therapy, which operate through cholinesterase inhibition or NMDA-receptor antagonism, Dimebon is a compound that seems to work through a novel mechanism by improving mitochondrial function in the brain. Moreover, Dimebon has been shown to be a promising therapeutic candidate in inhibiting brain cell death in both AD and HD preclinical models (Ref. 103).

Enhancing cellular defence mechanisms against different kinds of stress could be an attractive therapeutic strategy for neurodegenerative diseases. In particular, induction of expression of molecular chaperones might reduce the formation of misfolded proteins and toxic aggregates. Geldanamycin, a natural substance that modulates Hsp90 function, was previously shown to induce a heat-shock response through the activation of heat shock factor 1 (HSF1); it also reduces polyQ aggregation in mammalian cells (Ref. 104) and suppresses $\alpha$-synuclein neurotoxicity in flies (Ref. 105). Geldanamycin derivatives are now being considered for the development of a potential drug treatment for neurodegenerative diseases that involve protein aggregation.

Recently, Whitworth and colleagues showed that the drug Rapamycin, which is used in some transplant patients to prevent immune rejection, protects cells against the damaging effects of two of the mutant genes that cause inherited forms of PD (Ref. 53). This is an interesting observation if one considers that the current pharmacological interventions for PD are designed to replace or mimic the effects of dopamine, rather than actually change the course of the condition.

\section{Research in progress and outstanding research questions}

Recent work has highlighted the importance of protein translational switches during times of cellular and environmental stress, and the role of $4 \mathrm{E}-\mathrm{BP}$ in this process. Upon dietary restriction, a translational switch to nuclearencoded mitochondrial genes occurs in a $4 \mathrm{E}-$ BP-dependent manner (Ref. 106). This suggests an important role for enhanced mitochondrial function in conditions of cellular stress. It remains to be determined whether a 4E-BPdependent rescue of mitochondrial dysfunction in models of PD is mechanistically linked to the translational activation of nuclear encoded mitochondrial genes. It has recently been shown that inhibition of cap-dependent translation via $4 \mathrm{E}-\mathrm{BP}$ rescues phenotypes related to parkinsonism (Ref. 53). In addition, the oxidation-sensitive PD protein DJ-1 binds to mRNAs of several mitochondrial genes, including 4E-BP, which are released under conditions of oxidative stress (Ref. 107). These observations suggest that cellular responses to oxidative stress might involve a general switch in protein translation that leads to the selective upregulation of mitochondrial proteins. The molecular dissection of pathways controlling expression of mitochondrial genes is proceeding in earnest and is likely to lead to a better understanding of novel mitochondriaprotection mechanisms. Such understanding will hopefully lead to a better knowledge of the disease processes involving mitochondrial dysfunction, as well as more efficient treatment avenues.

Finally, one other potential route for treatment of neurodegenerative disease is the drug Metformin, which is already in common use for the treatment of type 2 diabetes, particularly in overweight and obese people. Metformin is also frequently used for research with the AMP analogue AICAR as an AMPK agonist. AMPK activation is one of the mechanisms by which mTOR can be suppressed, and studies that implicate AMPK in the regulation of mTOR

Accession information: doi:10.1017/S1462399410001456; Vol. 12; e12; April 2010 (c) Cambridge University Press 2010. Re-use permitted under a Creative Commons Licence - by-nc-sa. 
showed that pharmacological activation of AMPK by AICAR causes a dramatic reduction of 4E-BP phosphorylation (Ref. 108). Given that both diabetes and PD are reported to have a deregulation of the mTOR pathway, and that Metformin is one of the most prescribed drugs in the world, it would be interesting to see whether patients receiving the drug have a reduced risk of developing PD.

\section{Acknowledgements}

Inês Pimenta de Castro is recipient of a $\mathrm{PhD}$ grant (SFRH/BD/41682/2007) from FCT Portugal. We thank our anonymous peer reviewers for their constructive comments on this article.

\section{References}

1 Lane, N. (2005) Power, Sex, Suicide: mitochondria and the meaning of life. Oxford University Press, Oxford

2 Chan, D.C. (2006) Mitochondria: dynamic organelles in disease, aging, and development. Cell 125, 1241-1252

3 Taylor, S.W., Fahy, E. and Ghosh, S.S. (2003) Global organellar proteomics. Trends in Biotechnology 21, 82-88

4 Wang, Y. and Bogenhagen, D.F. (2006) Human mitochondrial DNA nucleoids are linked to protein folding machinery and metabolic enzymes at the mitochondrial inner membrane. Journal of Biological Chemistry 281, 25791-25802

5 Twig, G., Hyde, B. and Shirihai, O.S. (2008) Mitochondrial fusion, fission and autophagy as a quality control axis: the bioenergetic view. Biochimica et Biophysica Acta 1777, 1092-1097

6 Hardie, D.G. (2007) AMPK and SNF1:Snuffing Out Stress. Cell Metabolism 6, 339-340

7 Laplante, M. and Sabatini, D.M. (2009) mTOR signaling at a glance. Journal of Cell Science 122, 3589-3594

8 Youle, R.J. and Strasser, A. (2008) The BCL-2 protein family: opposing activities that mediate cell death. Nature Reviews Molecular Cell Biology 9, 47-59

9 Hamann, A., Brust, D. and Osiewacz, H.D. (2007) Deletion of putative apoptosis factors leads to lifespan extension in the fungal ageing model Podospora anserina. Molecular Microbiology 65, 948-958

10 Mattson, M.P. (2007) Calcium and neurodegeneration. Aging Cell 6, 337-350

11 Mattson, M.P., Gleichmann, M. and Cheng, A. (2008) Mitochondria in neuroplasticity and neurological disorders. Neuron 60, 748-766
12 Schapira, A.H. et al. (1989) Mitochondrial complex I deficiency in Parkinson's disease. Lancet 1, 1269

13 Swerdlow, R.H. et al. (1996) Origin and functional consequences of the complex I defect in Parkinson's disease. Annals of Neurology 40, 663-671

14 Navarro, A. and Boveris, A. (2009) Brain mitochondrial dysfunction and oxidative damage in Parkinson's disease. Journal of Bioenergetics and Biomembranes 41, 517-521

15 Langston, J.W. and Ballard, P.A. Jr (1983) Parkinson's disease in a chemist working with 1-methyl-4-phenyl-1,2,5,6-tetrahydropyridine. New England Journal of Medicine 309, 310

16 Gerlach, M. et al. (1991) MPTP mechanisms of neurotoxicity and their implications for Parkinson's disease. European Journal of Pharmacology 208, 273-286

17 Di Monte, D.A. (2003) The environment and Parkinson's disease: is the nigrostriatal system preferentially targeted by neurotoxins? Lancet Neurology 2, 531-538

18 Bogaerts, V. et al. (2008) Genetic variability in the mitochondrial serine protease HTRA2 contributes to risk for Parkinson disease. Human Mutation 29, 832-840

19 Valente, E.M. et al. (2004) PINK1 mutations are associated with sporadic early-onset parkinsonism. Annals of Neurology 56, 336-341

20 Ashley, A.K. et al. (2009) Analysis of targeted mutation in DJ-1 on cellular function in primary astrocytes. Toxicology Letters 184, 186-191

21 Canet-Aviles, R.M. et al. (2004) The Parkinson's disease protein DJ-1 is neuroprotective due to cysteine-sulfinic acid-driven mitochondrial localization. Proceedings of the National Academy of Sciences of the United States of America 101, 9103-9108

22 Junn, E. et al. (2009) Mitochondrial localization of DJ-1 leads to enhanced neuroprotection. Journal of Neuroscience Research 87, 123-129

23 Hayashi, T. et al. (2009) DJ-1 binds to mitochondrial complex I and maintains its activity. Biochemical and Biophysical Research Communications 3390, 667-672

24 Poole, A.C. et al. (2008) The PINK1/Parkin pathway regulates mitochondrial morphology. Proceedings of the National Academy of Sciences of the United States of America 105, 1638-1643

25 Deng, H. et al. (2008) The Parkinson's disease genes pink1 and parkin promote mitochondrial fission and/or inhibit fusion in Drosophila. Proceedings of the National Academy of Sciences of the United States of America 105, 14503-14508

Accession information: doi:10.1017/S1462399410001456; Vol. 12; e12; April 2010

(c) Cambridge University Press 2010. Re-use permitted under a Creative Commons Licence - by-nc-sa. 
26 Yang, Y. et al. (2008) Pink1 regulates mitochondrial dynamics through interaction with the fission/ fusion machinery. Proceedings of the National Academy of Sciences of the United States of America 105, 7070-7075

27 Dagda, R.K. et al. (2009) Loss of PINK1 function promotes mitophagy through effects on oxidative stress and mitochondrial fission. Journal of Biological Chemistry 284, 13843-13855

28 Narendra, D. et al. (2008) Parkin is recruited selectively to impaired mitochondria and promotes their autophagy. Journal of Cell Biology $183,795-803$

29 Plun-Favreau, H. et al. (2007) The mitochondrial protease HtrA2 is regulated by Parkinson's disease-associated kinase PINK1. Nature Cell Biology 9, 1243-1252

30 Li, X.J., Orr, A.L. and Li, S. (2009) Impaired mitochondrial trafficking in Huntington's disease. Biochimica et Biophysica Acta 1802, 62-65

31 Nakamura, K. et al. (2008) Optical reporters for the conformation of alpha-synuclein reveal a specific interaction with mitochondria. Journal of Neuroscience 28, 12305-12317

32 Bueler, H. (2009) Impaired mitochondrial dynamics and function in the pathogenesis of Parkinson's disease. Experimental Neurology 218, 235-246

33 Westermann, B. (2008) Molecular machinery of mitochondrial fusion and fission. Journal of Biological Chemistry 283, 13501-13505

34 Sandebring, A. et al. (2009) Mitochondrial alterations in PINK1 deficient cells are influenced by calcineurin-dependent dephosphorylation of dynamin-related protein 1. PLoS One 4, e5701

35 Park, J. et al. (2006) Mitochondrial dysfunction in Drosophila PINK1 mutants is complemented by parkin. Nature 441, 1157-1161

36 Clark, I.E. et al. (2006) Drosophila pink1 is required for mitochondrial function and interacts genetically with parkin. Nature 441, 1162-1166

37 Exner, N. et al. (2007) Loss-of-function of human PINK1 results in mitochondrial pathology and can be rescued by parkin. Journal of Neuroscience 27, 12413-12418

38 Lutz, A.K. et al. (2009) Loss of parkin or PINK1 function increases Drp1-dependent mitochondrial fragmentation. Journal of Biological Chemistry 284, 22938-22951

39 Weihofen, A. et al. (2009) Pink1 forms a multiprotein complex with Miro and Milton, linking Pink1 function to mitochondrial trafficking. Biochemistry 48, 2045-2052
40 Kim, Y. et al. (2008) PINK1 controls mitochondrial localization of Parkin through direct phosphorylation. Biochemical and Biophysical Research Communications 377, 975-980

41 Zhou, W. and Freed, C.R. (2005) DJ-1 up-regulates glutathione synthesis during oxidative stress and inhibits A53T alpha-synuclein toxicity. Journal of Biological Chemistry 280, 43150-43158

42 Abou-Sleiman, P.M. et al. (2006) A heterozygous effect for PINK1 mutations in Parkinson's disease? Annals of Neurology 60, 414-419

43 McBride, H.M. (2008) Parkin mitochondria in the autophagosome. Journal of Cell Biology 183, 757-759

44 Pridgeon, J.W. et al. (2007) PINK1 protects against oxidative stress by phosphorylating mitochondrial chaperone TRAP1. PLoS Biology 5, e172

45 Poon, H.F. et al. (2005) Mitochondrial associated metabolic proteins are selectively oxidized in A30P alpha-synuclein transgenic mice-a model of familial Parkinson's disease. Neurobiology of Disease 18, 492-498

46 Martin, L.J. et al. (2006) Parkinson's disease alphasynuclein transgenic mice develop neuronal mitochondrial degeneration and cell death. Journal of Neuroscience 26, 41-50

47 Ellis, C.E. et al. (2005) Mitochondrial lipid abnormality and electron transport chain impairment in mice lacking alpha-synuclein. Molecular and Cellular Biology 25, 10190-10201

48 Liu, W. et al. (2009) PINK1 defect causes mitochondrial dysfunction, proteasomal deficit and alpha-synuclein aggregation in cell culture models of Parkinson's disease. PLoS One 4, e4597

49 Todd, A.M. and Staveley, B.E. (2008) Pink1 suppresses alpha-synuclein-induced phenotypes in a Drosophila model of Parkinson's disease. Genome 51, 1040-1046

50 Shendelman, S. et al. (2004) DJ-1 is a redoxdependent molecular chaperone that inhibits alpha-synuclein aggregate formation. PLoS Biology 2, e362

51 Batelli, S. et al. (2008) DJ-1 modulates alphasynuclein aggregation state in a cellular model of oxidative stress: relevance for Parkinson's disease and involvement of HSP70. PLoS One 3, e1884

52 Xiong, H. et al. (2009) Parkin, PINK1, and DJ-1 form a ubiquitin E3 ligase complex promoting unfolded protein degradation. Journal of Clinical Investigation 119, 650-660

53 Tain, L.S. et al. (2009) Rapamycin activation of 4EBP prevents parkinsonian dopaminergic neuron loss. Nature Neuroscience 12, 1129-1135 
54 Venderova, K. et al. (2009) Leucine-rich repeat kinase 2 interacts with Parkin, DJ-1 and PINK-1 in a Drosophila melanogaster model of Parkinson's disease. Human Molecular Genetics 18, 4390-4404

55 Jaleel, M. et al. (2007) LRRK2 phosphorylates moesin at threonine-558: characterization of how Parkinson's disease mutants affect kinase activity. Biochemical Journal 405, 307-317

56 Clemens, M.J. (2001) Translational regulation in cell stress and apoptosis. Roles of the eIF4E binding proteins. Journal of Cellular and Molecular Medicine 5, 221-239

57 Reddy, P.H. and McWeeney, S. (2006) Mapping cellular transcriptosomes in autopsied Alzheimer's disease subjects and relevant animal models. Neurobiology of Aging 27, 1060-1077

58 Thinakaran, G. (1999) The role of presenilins in Alzheimer's disease. Journal of Clinical Investigation 104, 1321-1327

59 Corder, E.H. et al. (1993) Gene dose of apolipoprotein E type 4 allele and the risk of Alzheimer's disease in late onset families. Science 261, 921-923

60 Harold, D. et al. (2009) Genome-wide association study identifies variants at CLU and PICALM associated with Alzheimer's disease. Nature Genetics 41, 1088-1093

61 Caspersen, C. et al. (2005) Mitochondrial Abeta: a potential focal point for neuronal metabolic dysfunction in Alzheimer's disease. FASEB Journal 19, 2040-2041

62 Parker, W.D. Jr (1991) Cytochrome oxidase deficiency in Alzheimer's disease. Annals of the New York Academy of Sciences 640, 59-64

63 Maurer, I., Zierz, S. and Moller, H.J. (2000) A selective defect of cytochrome c oxidase is present in brain of Alzheimer disease patients. Neurobiology of Aging 21, 455-462

64 Casley, C.S. et al. (2002) Beta-amyloid fragment 25-35 causes mitochondrial dysfunction in primary cortical neurons. Neurobiology of Disease 10, 258-267

65 Boitier, E., Rea, R. and Duchen, M.R. (1999) Mitochondria exert a negative feedback on the propagation of intracellular $\mathrm{Ca} 2+$ waves in rat cortical astrocytes. Journal of Cell Biology 145, 795-808

66 Walsh, D.M. and Selkoe, D.J. (2007) A beta oligomers - a decade of discovery. Journal of Neurochemistry 101, 1172-1184

$67 \mathrm{Du}, \mathrm{H}$. et al. (2008) Cyclophilin D deficiency attenuates mitochondrial and neuronal perturbation and ameliorates learning and memory in Alzheimer's disease. Nature Medicine 14, 1097-1105

68 Anandatheerthavarada, H.K. et al. (2003)

Mitochondrial targeting and a novel

transmembrane arrest of Alzheimer's amyloid precursor protein impairs mitochondrial function in neuronal cells. Journal of Cell Biology $161,41-54$

69 Janus, C. et al. (2000) A beta peptide immunization reduces behavioural impairment and plaques in a model of Alzheimer's disease. Nature 408, 979-982

70 Dodart, J.C. et al. (2002) Immunization reverses memory deficits without reducing brain Abeta burden in Alzheimer's disease model. Nature Neuroscience 5, 452-457

71 Kotilinek, L.A. et al. (2002) Reversible memory loss in a mouse transgenic model of Alzheimer's disease. Journal of Neuroscience 22, 6331-6335

72 Park, H.J. et al. (2006) Beta-amyloid precursor protein is a direct cleavage target of HtrA2 serine protease. Implications for the physiological function of HtrA2 in the mitochondria. Journal of Biological Chemistry 281, 34277-34287

73 Falkevall, A. et al. (2006) Degradation of the amyloid beta-protein by the novel mitochondrial peptidasome, PreP. Journal of Biological Chemistry 281, 29096-29104

74 Bezprozvanny, I. and Mattson, M.P. (2008) Neuronal calcium mishandling and the pathogenesis of Alzheimer's disease. Trends in Neurosciences 31, 454-463

75 Verstreken, P. et al. (2005) Synaptic mitochondria are critical for mobilization of reserve pool vesicles at Drosophila neuromuscular junctions. Neuron 47, 365-378

76 Scheff, S.W. et al. (2006) Hippocampal synaptic loss in early Alzheimer's disease and mild cognitive impairment. Neurobiology of Aging 27, 1372-1384

77 Stokin, G.B. et al. (2005) Axonopathy and transport deficits early in the pathogenesis of Alzheimer's disease. Science 307, 1282-1288

78 Sze, C.I. et al. (1997) Loss of the presynaptic vesicle protein synaptophysin in hippocampus correlates with cognitive decline in Alzheimer disease. Journal of Neuropathology and Experimental Neurology 56, 933-944

79 Masliah, E. et al. (2001) Altered expression of synaptic proteins occurs early during progression of Alzheimer's disease. Neurology 56, 127-129

80 Reddy, P.H., Mao, P. and Manczak, M. (2009) Mitochondrial structural and functional dynamics in Huntington's disease. Brain Research Rev 61, $33-48$

Accession information: doi:10.1017/S1462399410001456; Vol. 12; e12; April 2010 (c) Cambridge University Press 2010. Re-use permitted under a Creative Commons Licence - by-nc-sa. 
81 [No authors listed] (1993) A novel gene containing a trinucleotide repeat that is expanded and unstable on Huntington's disease chromosomes. The Huntington's Disease Collaborative Research Group. Cell 72, 971-983

82 Bossy-Wetzel, E., Petrilli, A. and Knott, A.B. (2008) Mutant huntingtin and mitochondrial dysfunction. Trends in Neurosciences 31, 609-616

83 Lin, M.T. and Beal, M.F. (2006) Mitochondrial dysfunction and oxidative stress in neurodegenerative diseases. Nature 443, 787-795

$84 \mathrm{Gu}$, M. et al. (1996) Mitochondrial defect in Huntington's disease caudate nucleus. Annals of Neurology 39, 385-389

85 Rubinsztein, D.C. (2002) Lessons from animal models of Huntington's disease. Trends in Genetics 18, 202-209

86 Orr, A.L. et al. (2008) N-terminal mutant huntingtin associates with mitochondria and impairs mitochondrial trafficking. Journal of Neuroscience 28, 2783-2792

87 Panov, A.V. et al. (2002) Early mitochondrial calcium defects in Huntington's disease are a direct effect of polyglutamines. Nature Neuroscience 5, 731-736

88 Fernandes, H.B. et al. (2007) Mitochondrial sensitivity and altered calcium handling underlie enhanced NMDA-induced apoptosis in YAC128 model of Huntington's disease. Journal of Neuroscience 27, 13614-13623

89 Choo, Y.S. et al. (2004) Mutant huntingtin directly increases susceptibility of mitochondria to the calcium-induced permeability transition and cytochrome c release. Human Molecular Genetics 13, 1407-1420

90 Bae, B.I. et al. (2005) p53 mediates cellular dysfunction and behavioral abnormalities in Huntington's disease. Neuron 47, 29-41

91 Cui, L. et al. (2006) Transcriptional repression of PGC-1alpha by mutant huntingtin leads to mitochondrial dysfunction and neurodegeneration. Cell 127, 59-69

92 Hollenbeck, P.J. and Saxton, W.M. (2005) The axonal transport of mitochondria. Journal of Cell Science 118, 5411-5419

93 Gauthier, L.R. et al. (2004) Huntingtin controls neurotrophic support and survival of neurons by enhancing BDNF vesicular transport along microtubules. Cell 118, 127-138

94 McGuire, J.R. et al. (2006) Interaction of Huntingtinassociated protein-1 with kinesin light chain: implications in intracellular trafficking in neurons. Journal of Biological Chemistry 281, 3552-3559
95 Colin, E. et al. (2008) Huntingtin phosphorylation acts as a molecular switch for anterograde/ retrograde transport in neurons. EMBO Journal 27, 2124-2134

96 Trushina, E. etal. (2004) Mutant huntingtin impairs axonal trafficking in mammalian neurons in vivo and in vitro. Molecular and Cellular Biology 24, 8195-8209

97 Wang, H. et al. (2009) Effects of overexpression of huntingtin proteins on mitochondrial integrity. Human Molecular Genetics 18, 737-752

98 Kaltenbach, L.S. et al. (2007) Huntingtin interacting proteins are genetic modifiers of neurodegeneration. PLoS Genetics 3, e82

99 Sarkar, S. and Rubinsztein, D.C. (2008) Huntington's disease: degradation of mutant huntingtin by autophagy. FEBS Journal 275, 4263-4270

100 Wenz, T. et al. (2008) Activation of the PPAR/PGC1alpha pathway prevents a bioenergetic deficit and effectively improves a mitochondrial myopathy phenotype. Cell Metabolism 8, 249-256

101 Bubber, P. et al. (2005) Mitochondrial abnormalities in Alzheimer brain: mechanistic implications. Annals of Neurology 57, 695-703

102 Craft, S. et al. (2000) Insulin effects on glucose metabolism, memory, and plasma amyloid precursor protein in Alzheimer's disease differ according to apolipoprotein-E genotype. Annals of the New York Academy of Sciences 903, 222-228

103 Moreira, P.I. et al. (2009) Mitochondria: A therapeutic target in neurodegeneration. Biochimica et Biophysica Acta 1802, 212-220

104 Sittler, A. et al. (2001) Geldanamycin activates a heat shock response and inhibits huntingtin aggregation in a cell culture model of Huntington's disease. Human Molecular Genetics 10, 1307-1315

105 Auluck, P.K., Meulener, M.C. and Bonini, N.M. (2005) Mechanisms of Suppression of \{alpha\}Synuclein Neurotoxicity by Geldanamycin in Drosophila. Journal of Biological Chemistry 280, 2873-2878

106 Zid, B.M. et al. (2009) 4E-BP extends lifespan upon dietary restriction by enhancing mitochondrial activity in Drosophila. Cell 139, 149-160

107 van der Brug, M.P. et al. (2008) RNA binding activity of the recessive parkinsonism protein DJ-1 supports involvement in multiple cellular pathways. Proceedings of the National Academy of Sciences of the United States of America 105, 10244-10249

108 Corradetti, M.N. and Guan, K.L. (2006) Upstream of the mammalian target of rapamycin: do all roads pass through mTOR? Oncogene 25, 6347-6360

Accession information: doi:10.1017/S1462399410001456; Vol. 12; e12; April 2010 (c) Cambridge University Press 2010. Re-use permitted under a Creative Commons Licence - by-nc-sa. 
109 Sandoval, H. et al. (2008) Essential role for Nix in autophagic maturation of erythroid cells. Nature 454, 232-235

110 Schweers, R.L. et al. (2007) NIX is required for programmed mitochondrial clearance during reticulocyte maturation. Proceedings of the National Academy of Sciences of the United States of America 104, 19500-19505

111 Kissova, I. et al. (2004) Uth1p is involved in the autophagic degradation of mitochondria. Journal of Biological Chemistry 279, 39068-39074

112 Tal, R. et al. (2007) Aup1p, a yeast mitochondrial protein phosphatase homolog, is required for efficient stationary phase mitophagy and cell survival. Journal of Biological Chemistry 282, 5617-5624

113 Chu, J. et al. (2009) A mouse forward genetics screen identifies LISTERIN as an E3 ubiquitin ligase involved in neurodegeneration. Proceedings of the National Academy of Sciences of the United States of America 106, 2097-2103

$114 \mathrm{Li}, \mathrm{W}$. et al. (2008) Genome-wide and functional annotation of human E3 ubiquitin ligases identifies MULAN, a mitochondrial E3 that regulates the organelle's dynamics and signaling. PLoS One 3, e1487

115 Geisler, S. et al. (2010) PINK1/Parkin-mediated mitophagy is dependent on VDAC1 and p62/ SQSTM1. Nature Cell Biology 12, 119-131

116 Kirkin, V. et al. (2009) A role for ubiquitin in selective autophagy. Molecular Cell 34, 259-269

117 Chao, J.R. et al. (2008) Hax1-mediated processing of HtrA2 by Parl allows survival of lymphocytes and neurons. Nature 452, 98-102

118 Jeyaraju, D.V. et al. (2009) Hax1 lacks BH modules and is peripherally associated to heavy membranes: implications for Omi/HtrA2 and PARL activity in the regulation of mitochondrial stress and apoptosis. Cell Death and Differentiation 16, 1622-1629

119 Moisoi, N. et al. (2009) Mitochondrial dysfunction triggered by loss of HtrA2 results in the activation of a brain-specific transcriptional stress response. Cell Death and Differentiation 16, 449-464

120 Valente, E.M. et al. (2004) Hereditary early-onset Parkinson's disease caused by mutations in PINK1. Science 304, 1158-1160

\section{Further reading, resources and contacts}

\section{Books}

Lane, N. (2005) Power, Sex, Suicide: Mitochondria and the Meaning of Life. OUP, Oxford

A provocative book full of interesting concepts, including links between ageing and neurodegenerative disease.

Gibson G.E., Ratan R.R. and Flint Beal M. eds (2008) Mitochondria and Oxidative Stress in Neurodegenerative Disorders. Annals of the New York Academy of Sciences

A combination of basic and clinical research to give the reader the most current information on aspects of mitochondrial function linked to age-related neurodegenerative diseases and their treatment.

\section{Websites}

A useful network of research, support and care in Alzheimer disease provided by a leading voluntary health organisation. It includes in the 'Clinical Studies' section an accurate description of the Phase III interventional trials currently in use:

http://www.alz.org

A database of Drosophila melanogaster nuclear genes encoding mitochondrial proteins can be found at: http://www2.ba.itb.cnr.it/MitoDrome

The Whitehead Institute Video Gallery has lectures from researchers at the Whitehead Institute. It includes several interesting lectures by Susan Lindquist on protein misfolding and neurodegenerative diseases as well as David Sabatini on growth control pathways:

www.wi.mit.edu/news/video_gallery

(continued on next page) 


\section{Further reading, resources and contacts (continued)}

The Encyclopedia of Neuroscience explores a wide variety of topics related to different areas of neuroscience. It is a very useful tool, well written and easily accessible:

www.sciencedirect.com/science/referenceworks/9780080450469

The Allen Mouse Brain Atlas is an interactive, genome-wide image database of gene expression. It also includes information regarding the cortex of the human brain:

www.mouse.brain-map.org

\section{Features associated with this article}

\section{Figures}

Figure 1. Core quality-control pathways in mitochondria.

Figure 2. Molecular determinants of mitochondrial quality-control networks.

Figure 3. Role of the major PD-associated genes in mitochondrial dysfunction.

Figure 4. Mitochondrial dysfunction in AD.

Figure 5. Role of Htt protein in mitochondrial trafficking and dynamics.

Table

Table 1. Genes involved in major mitochondria-associated neurodegenerative diseases.

\section{Citation details for this article}

Inês Pimenta de Castro, L. Miguel Martins and Roberta Tufi (2010) Mitochondrial quality control and neurological disease: an emerging connection. Expert Rev. Mol. Med. Vol. 12, e12, April 2010, doi:10.1017/S1462399410001456 